\title{
Urban Myth and Reality: The State of Urban Growth and Development in Assam
}

\author{
Rakesh Chetry ${ }^{1}$, Dr. Bimal K. Kar ${ }^{2}$ \\ ${ }^{1}$ (Research Scholar, Department of Geography, Gauhati University, India) \\ 2(Professor and Head, Department of Geography, Gauhati University, India)
}

\begin{abstract}
An urban centre, being a living dynamic entity, has the capacity to support outsized human population with great potential for an improved quality of urban life. But, contrary to the developed countries of the world, the phenomenon of urbanisation and urban development relationship can be hardly noticed in developing economies like India. Assam being no exception has been experiencing a phenomenal urban growth with absolute increase in number of urban centres (12 to 214) and urban population (77 thousand to 4.4 million) during 1901-2011. But, it is discouraging that the rate of urban development as compared to the urban growth in the state is quite low. Therefore, it is still unclear, whether urbanisation in Assam is the product of urban development or it is mere reflection of high population increase and consequent unbalanced urban development in urban centres. Based primarily on Secondary data, the study examines the level of urbanisation in Assam with respect to urban development including the demographic and social implications of rapidly growing urban population.
\end{abstract}

Keywords: Urban development, Urban growth, Urbanisation

\section{INTRODUCTION:}

Urbanization and Urban Development are two significant facets of urban systems, where both exercise a reciprocal cause-effect relationship. However, in developed economies, it is the economic development which gears up the urbanization process, whereas in developing economies like India, large scale rural-urban migration and agglomeration of urban settlements result in increasing urbanisation level and urban intensity. Thus urbanisation is in itself a complex phenomenon where the main point to judge urbanisation is population. Initially a country's development was based on its degree of urbanisation irrespective of the adequate availability of amenities, the urban centre provides to its inhabitants. Thus Indian cities experienced urbanisation only in terms of population concentration and not in terms of urban development. Ultimately the outcome of this trend is the non-availability of bare minimum household and institutional services to the urban dwellers, who although are living in million plus cities in India, but lack a decent standard of living. Therefore urbanisation and urban development are two conflicting terms with respect to India including Assam as a whole.

Assam, which lies in the North East corner of India ranks first in terms of population and second in terms of area with respect to the other neighbouring states of the region. It is noteworthy that Assam alone holds more than 52 per cent of the total urban population of North East India including Sikkim. Accordingly the urban population of Assam is much more than the combined urban population of other north-eastern states. However in terms of percentage of urban population to total population, it is the least urbanised state (14.1 per cent) as compared to its other associates. So far the trend of urban growth in the state is concerned, its number of urban centres has increased from 12 to 214 (including Census towns) during 1901-2011 with consequent population increase from 77 thousand to 43.88 lakhs. This absolute increase in the number of urban people which is often referred to as urban growth has indeed been remarkable. However, one wonders, if this fast increasing size of the urban areas is really the result of urban development and, therefore can truly be called as urbanization, or it is merely a reflection of high population increase and an unbalanced economic growth. Adding to the woes of urban scenario in the state, there exists unbalanced urban development among the districts. Nearly 51 per cent of the urban population of the state is concentrated in the five districts, viz. Kamrup Metropolitan, Nagaon, Cachar, Tinsukia and Dibrugarh. Similarly, there exists significant spatial variation in respect of urban population growth during 2001-11 as against the state average of 27.61 per cent. Thus, such a high and spatio-temporal variation in the urban setting of the region is likely to pressurize the amenities and services provided to the urban dwellers and adversely affect the urban development scenario of the region. With the above background, an attempt is made to analyse the spatio-temporal variation in growth and distribution of urban population in the state with respect to the country as a whole, to examine the spatial level of urbanisation and urban intensity in the state and to access the change in level of urban development in the light of urbanisation and urban intensity of Assam since 1991. 

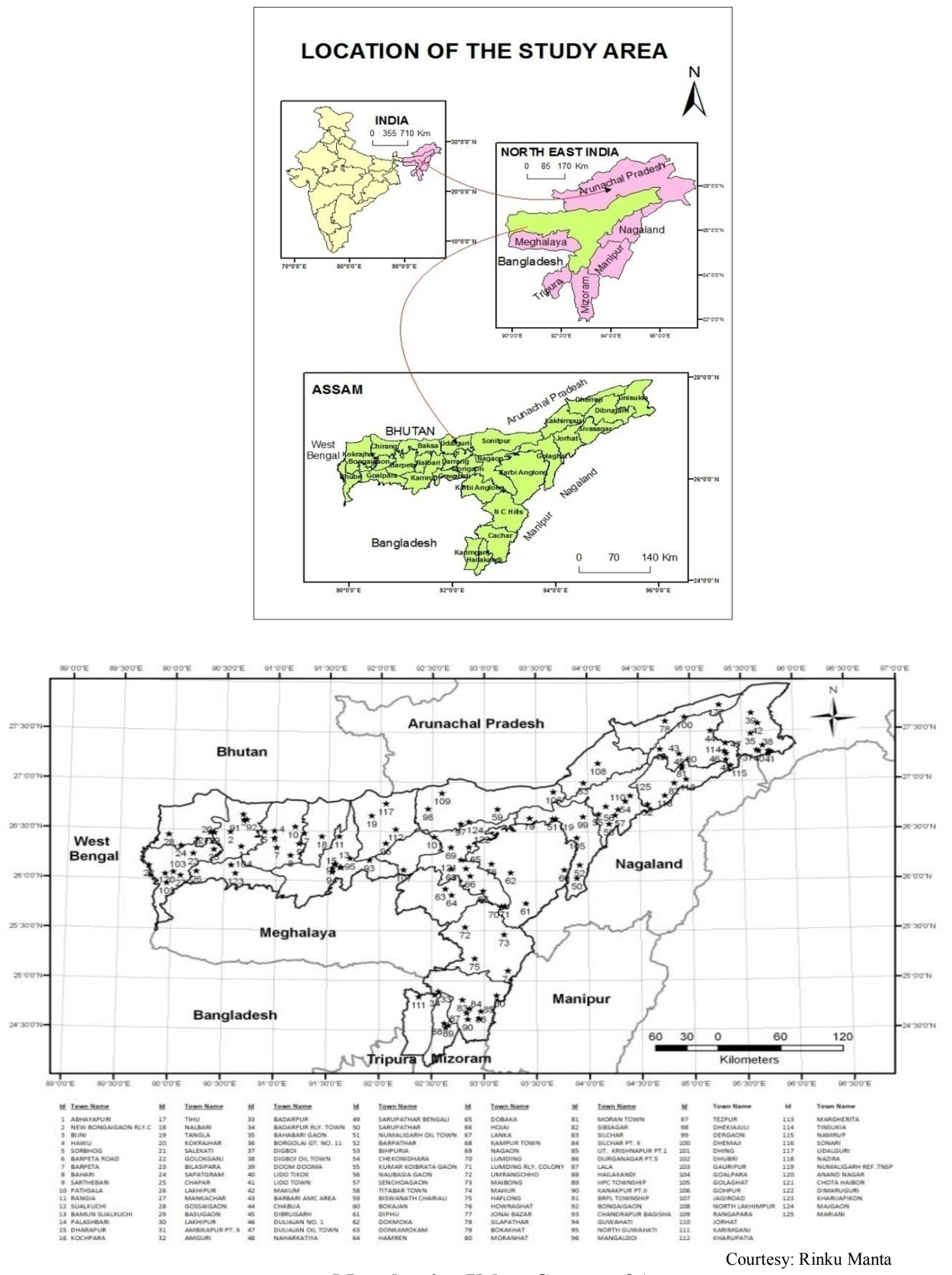

Map showing Urban Centres of Assam

II. Database And Methodology

Almost all the required data for computation of Urbanisation, Urban Intensity and Urban Development has been acquired from different Census of India publications for the period 1951-2011 in general and 19712011 in particular. Town Directory of Assam since 1971 has also been utilized for collection of urban-centre wise data of different aspects. Moreover district level data of the indicators adopted to analyse the urban development of the state has been derived from Household Amenities Booklet published by the Census of India 
itself. Besides Statistical Handbook of Assam, Economic Survey of Assam, newspapers and journals have been considered for further analyses.

The data so collected has been arranged, tabulated and verified accordingly. The tabulated data has been processed by using appropriate quantitative techniques ranging from simple percentage method to complex Principal Component Analysis (PCA) for computing the level of urbanisation and urban development in the region. Various indexes have been worked out to gauge the urban phenomena of Assam properly. Among these, Urban Intensity Index has been computed by using the average of four indicators viz. percentage of urban centres, urban population, urban density and urban growth rate. Similarly to find out the urbanisation level, PCA of four indices i.e. average spacing of urban centres, average size of urban area, urban density of population including proportion of urban population has been adopted. Likewise to analyse the level of urban development altogether ten indicators viz. literacy rate, proportion of non-agricultural workers, percentage of urban households with electricity, tap water, hand pump, latrines, LPG as cooking fuel, number of hotels and guest houses for tourists, educational and medical institutions has been employed and PCA has been adopted for garnering out the exact scenario. For clear exposition of the problem, the computed data have been properly represented through graphs, diagrams and maps drawn with the help of SPSS and GIS.

\section{Analysis And Discussion}

\subsection{District wise Trend of Urban Population and Growth in Assam, 1961-2011}

The overall trend of urban population of Assam reveals steady urban growth rate from 1901 to 1941, where the urban growth rate never exceeded more than 37 per cent. Conversely, the decades from 1951-1991 registered rapid increase in urban population with growth rate always above 65 per cent. Most remarkably the 1961 census experienced 126.57 per cent urban growth rate which is the highest in demographic history of Assam. However since 1991 till date the urban growth rate is showing a declining trend, reaching to a record lowest of 27.6 per cent in 2011. Thus although the growth rate of urban population in the state of Assam has been checked to a certain extent in recent time but its contribution towards region's overall urban population growth is still found to be very significant owing to large urban population base.

TABLE 1: Spatial variation in the Urban Growth pattern of Assam, 1961-2011

\begin{tabular}{|c|c|c|c|c|c|c|c|c|c|c|c|c|c|c|c|c|c|c|c|c|}
\hline \multirow{2}{*}{$\begin{array}{l}\text { Distric } \\
\text { ts }\end{array}$} & \multicolumn{4}{|c|}{1961} & \multicolumn{4}{|c|}{1971} & \multicolumn{4}{|c|}{1991} & \multicolumn{4}{|c|}{2001} & \multicolumn{4}{|c|}{2011} \\
\hline & I & II & III & $\begin{array}{l}\mathbf{I} \\
\text { V }\end{array}$ & I & II & III & $\begin{array}{l}\mathbf{I} \\
\text { V }\end{array}$ & I & II & III & $\begin{array}{l}\mathbf{I} \\
\text { V }\end{array}$ & I & II & III & $\begin{array}{l}\text { I } \\
\text { V }\end{array}$ & I & II & III & $\begin{array}{l}\text { I } \\
\text { V }\end{array}$ \\
\hline $\begin{array}{l}\text { Kokra } \\
\text { jhar }\end{array}$ & $\begin{array}{l}948 \\
9 \\
\end{array}$ & $\begin{array}{l}1.2 \\
1 \\
\end{array}$ & & 1 & $\begin{array}{l}1706 \\
0\end{array}$ & $\begin{array}{l}3.7 \\
6 \\
\end{array}$ & $\begin{array}{l}6.0 \\
4 \\
\end{array}$ & 1 & $\begin{array}{l}5072 \\
4 \\
\end{array}$ & $\begin{array}{l}6.3 \\
3 \\
\end{array}$ & $\begin{array}{l}5.5 \\
9 \\
\end{array}$ & 3 & $\begin{array}{l}5150 \\
1\end{array}$ & $\begin{array}{l}7.0 \\
6 \\
\end{array}$ & $\begin{array}{l}0.1 \\
5 \\
\end{array}$ & 4 & $\begin{array}{l}5494 \\
1\end{array}$ & $\begin{array}{l}6.1 \\
9 \\
\end{array}$ & $\begin{array}{l}1.0 \\
6 \\
\end{array}$ & 4 \\
\hline $\begin{array}{l}\text { Dhubr } \\
\text { i }\end{array}$ & $\begin{array}{l}649 \\
72 \\
\end{array}$ & $\begin{array}{l}8.3 \\
2 \\
\end{array}$ & $\begin{array}{l}7.8 \\
8 \\
\end{array}$ & 5 & $\begin{array}{l}9322 \\
0 \\
\end{array}$ & $\begin{array}{l}10 . \\
95\end{array}$ & $\begin{array}{l}3.6 \\
8 \\
\end{array}$ & 5 & $\begin{array}{l}1619 \\
81\end{array}$ & $\begin{array}{l}12 . \\
16\end{array}$ & $\begin{array}{l}2.8 \\
0 \\
\end{array}$ & 7 & $\begin{array}{l}1924 \\
43\end{array}$ & $\begin{array}{l}11 . \\
75\end{array}$ & $\begin{array}{l}1.7 \\
4\end{array}$ & 8 & $\begin{array}{l}2037 \\
01\end{array}$ & $\begin{array}{l}10 . \\
45\end{array}$ & $\begin{array}{l}0.5 \\
7 \\
\end{array}$ & 9 \\
\hline $\begin{array}{l}\text { Goalp } \\
\text { ara }\end{array}$ & $\begin{array}{l}136 \\
92 \\
\end{array}$ & $\begin{array}{l}1.7 \\
5\end{array}$ & $\begin{array}{l}2.9 \\
9 \\
\end{array}$ & 1 & $\begin{array}{l}2203 \\
5\end{array}$ & $\begin{array}{l}5.0 \\
8 \\
\end{array}$ & $\begin{array}{l}4.8 \\
7 \\
\end{array}$ & 2 & $\begin{array}{l}5209 \\
6 \\
\end{array}$ & $\begin{array}{l}7.8 \\
2 \\
\end{array}$ & $\begin{array}{l}4.4 \\
0\end{array}$ & 2 & $\begin{array}{l}6690 \\
2 \\
\end{array}$ & $\begin{array}{l}8.1 \\
4\end{array}$ & $\begin{array}{l}2.5 \\
3 \\
\end{array}$ & 3 & $\begin{array}{l}1380 \\
62\end{array}$ & $\begin{array}{l}13 . \\
69\end{array}$ & $\begin{array}{l}7.5 \\
1 \\
\end{array}$ & $\begin{array}{l}1 \\
1 \\
\end{array}$ \\
\hline $\begin{array}{l}\text { Barpet } \\
\text { a }\end{array}$ & $\begin{array}{l}373 \\
17 \\
\end{array}$ & $\begin{array}{l}4.7 \\
8 \\
\end{array}$ & $\begin{array}{l}5.8 \\
5 \\
\end{array}$ & 3 & $\begin{array}{l}6872 \\
8 \\
\end{array}$ & $\begin{array}{l}7.0 \\
9 \\
\end{array}$ & $\begin{array}{l}6.2 \\
9 \\
\end{array}$ & 6 & $\begin{array}{l}9731 \\
8 \\
\end{array}$ & $\begin{array}{l}7.0 \\
2 \\
\end{array}$ & $\begin{array}{l}1.7 \\
5\end{array}$ & 7 & $\begin{array}{l}1268 \\
68 \\
\end{array}$ & $\begin{array}{l}7.7 \\
0\end{array}$ & $\begin{array}{l}2.6 \\
9 \\
\end{array}$ & 7 & $\begin{array}{l}1473 \\
53\end{array}$ & $\begin{array}{l}8.7 \\
0 \\
\end{array}$ & $\begin{array}{l}1.5 \\
1 \\
\end{array}$ & 9 \\
\hline $\begin{array}{l}\text { Morig } \\
\text { aon }\end{array}$ & -- & & & & -- & & -- & & $\begin{array}{l}3298 \\
9 \\
\end{array}$ & $\begin{array}{l}5.1 \\
5 \\
\end{array}$ & -- & 3 & $\begin{array}{l}3798 \\
8 \\
\end{array}$ & $\begin{array}{l}4.8 \\
9 \\
\end{array}$ & $\begin{array}{l}1.4 \\
2 \\
\end{array}$ & 2 & $\begin{array}{l}7329 \\
8 \\
\end{array}$ & $\begin{array}{l}7.6 \\
6 \\
\end{array}$ & $\begin{array}{l}6.7 \\
9 \\
\end{array}$ & 6 \\
\hline $\begin{array}{l}\text { Nagao } \\
\text { n }\end{array}$ & $\begin{array}{l}812 \\
17 \\
\end{array}$ & $\begin{array}{l}10 . \\
39 \\
\end{array}$ & $\begin{array}{l}6.4 \\
3 \\
\end{array}$ & 4 & $\begin{array}{l}1193 \\
37 \\
\end{array}$ & $\begin{array}{l}9.5 \\
3 \\
\end{array}$ & $\begin{array}{l}3.9 \\
2 \\
\end{array}$ & 4 & $\begin{array}{l}2057 \\
22 \\
\end{array}$ & $\begin{array}{l}10 . \\
87 \\
\end{array}$ & $\begin{array}{l}2.7 \\
6 \\
\end{array}$ & 7 & $\begin{array}{l}2782 \\
87 \\
\end{array}$ & $\begin{array}{l}12 . \\
02\end{array}$ & $\begin{array}{l}3.0 \\
7 \\
\end{array}$ & $\begin{array}{l}1 \\
\mathbf{0}\end{array}$ & $\begin{array}{l}3695 \\
34 \\
\end{array}$ & $\begin{array}{l}13 . \\
09\end{array}$ & $\begin{array}{l}2.8 \\
8 \\
\end{array}$ & $\begin{array}{l}1 \\
6 \\
\end{array}$ \\
\hline $\begin{array}{l}\text { Sonitp } \\
\text { ur }\end{array}$ & $\begin{array}{l}305 \\
22\end{array}$ & $\begin{array}{l}3.9 \\
1\end{array}$ & $\begin{array}{l}4.9 \\
2\end{array}$ & 2 & $\begin{array}{l}7157 \\
3\end{array}$ & $\begin{array}{l}7.8 \\
9\end{array}$ & $\begin{array}{l}8.9 \\
0\end{array}$ & 4 & $\begin{array}{l}1039 \\
08\end{array}$ & $\begin{array}{l}7.3 \\
0\end{array}$ & $\begin{array}{l}1.8 \\
8\end{array}$ & 4 & $\begin{array}{l}1757 \\
94\end{array}$ & $\begin{array}{l}10 . \\
45\end{array}$ & $\begin{array}{l}5.4 \\
0\end{array}$ & 6 & $\begin{array}{l}1738 \\
45\end{array}$ & $\begin{array}{l}9.0 \\
3\end{array}$ & $\begin{array}{l}- \\
0.1 \\
1\end{array}$ & 8 \\
\hline $\begin{array}{l}\text { Lakhi } \\
\text { mpur }\end{array}$ & $\begin{array}{l}977 \\
4\end{array}$ & $\begin{array}{l}1.2 \\
5\end{array}$ & $\begin{array}{l}12 . \\
19\end{array}$ & 2 & $\begin{array}{l}2535 \\
0\end{array}$ & $\begin{array}{l}5.2 \\
7\end{array}$ & $\begin{array}{l}10 . \\
00\end{array}$ & 2 & $\begin{array}{l}4913 \\
0\end{array}$ & $\begin{array}{l}6.5 \\
3\end{array}$ & $\begin{array}{l}3.3 \\
6\end{array}$ & 2 & $\begin{array}{l}6515 \\
3\end{array}$ & $\begin{array}{l}7.3 \\
3\end{array}$ & $\begin{array}{l}2.8 \\
6\end{array}$ & 2 & $\begin{array}{l}9133 \\
3\end{array}$ & $\begin{array}{l}8.7 \\
6\end{array}$ & $\begin{array}{l}3.4 \\
3\end{array}$ & 4 \\
\hline $\begin{array}{l}\text { Dhem } \\
\text { aji }\end{array}$ & --- & & & & -- & & -- & & 8891 & $\begin{array}{l}1.8 \\
6 \\
\end{array}$ & -- & 1 & $\begin{array}{l}3883 \\
2\end{array}$ & $\begin{array}{l}6.7 \\
9 \\
\end{array}$ & $\begin{array}{l}15 . \\
88\end{array}$ & 3 & $\begin{array}{l}4828 \\
5\end{array}$ & $\begin{array}{l}7.0 \\
4\end{array}$ & $\begin{array}{l}2.2 \\
0 \\
\end{array}$ & 4 \\
\hline $\begin{array}{l}\text { Tinsuk } \\
\text { ia }\end{array}$ & $\begin{array}{l}716 \\
88 \\
\end{array}$ & $\begin{array}{l}9.1 \\
7 \\
\end{array}$ & $\begin{array}{l}13 . \\
39\end{array}$ & 4 & $\begin{array}{l}1130 \\
51\end{array}$ & $\begin{array}{l}17 . \\
27\end{array}$ & $\begin{array}{l}4.6 \\
6 \\
\end{array}$ & 6 & $\begin{array}{l}1586 \\
74 \\
\end{array}$ & $\begin{array}{l}16 . \\
48\end{array}$ & $\begin{array}{l}1.7 \\
1 \\
\end{array}$ & 6 & $\begin{array}{l}\mathbf{2 2 3 9} \\
\mathbf{5 7} \\
\end{array}$ & $\begin{array}{l}19 . \\
47\end{array}$ & $\begin{array}{l}3.5 \\
1 \\
\end{array}$ & $\begin{array}{l}1 \\
0\end{array}$ & $\begin{array}{l}2647 \\
43 \\
\end{array}$ & $\begin{array}{l}19 . \\
94\end{array}$ & $\begin{array}{l}1.6 \\
9 \\
\end{array}$ & $\begin{array}{l}1 \\
3 \\
\end{array}$ \\
\hline $\begin{array}{l}\text { Dibru } \\
\text { garh }\end{array}$ & $\begin{array}{l}698 \\
90 \\
\end{array}$ & $\begin{array}{l}8.9 \\
4 \\
\end{array}$ & $\begin{array}{l}6.2 \\
8 \\
\end{array}$ & 3 & $\begin{array}{l}1144 \\
79 \\
\end{array}$ & $\begin{array}{l}15 . \\
13\end{array}$ & $\begin{array}{l}5.0 \\
6 \\
\end{array}$ & 5 & $\begin{array}{l}1835 \\
80 \\
\end{array}$ & $\begin{array}{l}17 . \\
61\end{array}$ & $\begin{array}{l}2.4 \\
0 \\
\end{array}$ & 6 & $\begin{array}{l}2284 \\
38 \\
\end{array}$ & $\begin{array}{l}19 . \\
27\end{array}$ & $\begin{array}{l}2.2 \\
1 \\
\end{array}$ & 9 & $\begin{array}{l}2437 \\
30 \\
\end{array}$ & $\begin{array}{l}18 . \\
37\end{array}$ & $\begin{array}{l}0.6 \\
5 \\
\end{array}$ & 9 \\
\hline $\begin{array}{l}\text { Sibsag } \\
\text { ar }\end{array}$ & $\begin{array}{l}200 \\
16\end{array}$ & $\begin{array}{l}2.5 \\
6\end{array}$ & $\begin{array}{l}3.0 \\
1\end{array}$ & 2 & $\begin{array}{l}4043 \\
4\end{array}$ & $\begin{array}{l}6.1 \\
8\end{array}$ & $\begin{array}{l}7.2 \\
8\end{array}$ & 5 & $\begin{array}{l}6568 \\
9\end{array}$ & $\begin{array}{l}7.2 \\
3 \\
\end{array}$ & $\begin{array}{l}2.4 \\
6\end{array}$ & 5 & $\begin{array}{l}9717 \\
9\end{array}$ & $\begin{array}{l}9.2 \\
4\end{array}$ & $\begin{array}{l}4.0 \\
0\end{array}$ & 5 & $\begin{array}{l}1100 \\
96\end{array}$ & $\begin{array}{l}9.5 \\
6\end{array}$ & $\begin{array}{l}1.2 \\
6\end{array}$ & 7 \\
\hline Jorhat & $\begin{array}{l}341 \\
88\end{array}$ & $\begin{array}{l}4.3 \\
7\end{array}$ & $\begin{array}{l}7.7 \\
8\end{array}$ & 2 & $\begin{array}{l}8572 \\
8\end{array}$ & $\begin{array}{l}13 . \\
09\end{array}$ & $\begin{array}{l}9.6 \\
3 \\
\end{array}$ & 2 & $\begin{array}{l}1330 \\
32\end{array}$ & $\begin{array}{l}15 . \\
26\end{array}$ & $\begin{array}{l}2.2 \\
2\end{array}$ & 3 & $\begin{array}{l}1713 \\
20\end{array}$ & $\begin{array}{l}17 . \\
14\end{array}$ & $\begin{array}{l}2.5 \\
6\end{array}$ & 7 & $\begin{array}{l}2205 \\
34\end{array}$ & $\begin{array}{l}20 . \\
19\end{array}$ & $\begin{array}{l}2.5 \\
6\end{array}$ & $\begin{array}{l}1 \\
1\end{array}$ \\
\hline $\begin{array}{l}\text { Golag } \\
\text { hat }\end{array}$ & $\begin{array}{l}225 \\
01 \\
\end{array}$ & $\begin{array}{l}2.8 \\
8 \\
\end{array}$ & $\begin{array}{l}10 . \\
51 \\
\end{array}$ & 2 & $\begin{array}{l}2856 \\
2 \\
\end{array}$ & $\begin{array}{l}5.4 \\
5 \\
\end{array}$ & $\begin{array}{l}2.4 \\
1 \\
\end{array}$ & 2 & $\begin{array}{l}4883 \\
8 \\
\end{array}$ & $\begin{array}{l}5.8 \\
9 \\
\end{array}$ & $\begin{array}{l}2.7 \\
2 \\
\end{array}$ & 3 & $\begin{array}{l}8113 \\
8 \\
\end{array}$ & $\begin{array}{l}8.5 \\
7 \\
\end{array}$ & $\begin{array}{l}5.2 \\
1 \\
\end{array}$ & 6 & $\begin{array}{l}9773 \\
6 \\
\end{array}$ & $\begin{array}{l}9.1 \\
6 \\
\end{array}$ & $\begin{array}{l}1.8 \\
8 \\
\end{array}$ & 7 \\
\hline $\begin{array}{l}\text { K.Ang } \\
\text { long }\end{array}$ & -- & & & & $\begin{array}{l}1020 \\
0\end{array}$ & $\begin{array}{l}2.6 \\
9 \\
\end{array}$ & -- & 1 & $\begin{array}{l}7046 \\
6 \\
\end{array}$ & $\begin{array}{l}10 . \\
63\end{array}$ & $\begin{array}{l}10 . \\
15\end{array}$ & 6 & $\begin{array}{l}9193 \\
0 \\
\end{array}$ & $\begin{array}{l}11 . \\
30\end{array}$ & $\begin{array}{l}2.6 \\
9 \\
\end{array}$ & 6 & $\begin{array}{l}1129 \\
66\end{array}$ & $\begin{array}{l}11 . \\
81\end{array}$ & $\begin{array}{l}2.0 \\
8 \\
\end{array}$ & 7 \\
\hline $\begin{array}{l}\text { D. } \\
\text { Hasao }\end{array}$ & $\begin{array}{l}326 \\
5 \\
\end{array}$ & $\begin{array}{l}0.4 \\
2 \\
\end{array}$ & $\begin{array}{l}4.1 \\
8 \\
\end{array}$ & 1 & 5197 & $\begin{array}{l}6.8 \\
3 \\
\end{array}$ & $\begin{array}{l}4.7 \\
6 \\
\end{array}$ & 1 & $\begin{array}{l}3448 \\
6 \\
\end{array}$ & $\begin{array}{l}22 . \\
86\end{array}$ & $\begin{array}{l}9.9 \\
2 \\
\end{array}$ & 3 & $\begin{array}{l}5943 \\
5 \\
\end{array}$ & $\begin{array}{l}31 . \\
60\end{array}$ & $\begin{array}{l}5.5 \\
9 \\
\end{array}$ & 4 & $\begin{array}{l}6248 \\
9 \\
\end{array}$ & $\begin{array}{l}29 . \\
19\end{array}$ & $\begin{array}{l}0.5 \\
0 \\
\end{array}$ & 4 \\
\hline $\begin{array}{l}\text { Cacha } \\
\mathbf{r}\end{array}$ & $\begin{array}{l}436 \\
26 \\
\end{array}$ & $\begin{array}{l}5.5 \\
8 \\
\end{array}$ & $\begin{array}{l}2.5 \\
1 \\
\end{array}$ & 2 & $\begin{array}{l}\mathbf{5 5 5 3} \\
7 \\
\end{array}$ & $\begin{array}{l}6.7 \\
4 \\
\end{array}$ & $\begin{array}{l}2.4 \\
4\end{array}$ & 2 & $\begin{array}{l}1192 \\
24 \\
\end{array}$ & $\begin{array}{l}9.8 \\
0 \\
\end{array}$ & $\begin{array}{l}3.8 \\
9 \\
\end{array}$ & 2 & $\begin{array}{l}2013 \\
87 \\
\end{array}$ & $\begin{array}{l}13 \\
09 \\
\end{array}$ & $\begin{array}{l}5.3 \\
8 \\
\end{array}$ & 7 & $\begin{array}{l}3154 \\
64 \\
\end{array}$ & $\begin{array}{l}18 . \\
16\end{array}$ & $\begin{array}{l}4.5 \\
9 \\
\end{array}$ & $\begin{array}{l}1 \\
9\end{array}$ \\
\hline
\end{tabular}


Urban Myth and Reality: The State of Urban Growth and Development In Assam

\begin{tabular}{|c|c|c|c|c|c|c|c|c|c|c|c|c|c|c|c|c|c|c|c|c|}
\hline & & & & & & & & & & & & & & 4 & & & & & & \\
\hline $\begin{array}{l}\text { Karim } \\
\text { ganj }\end{array}$ & $\begin{array}{l}345 \\
68 \\
\end{array}$ & $\begin{array}{l}4.4 \\
2 \\
\end{array}$ & $\begin{array}{l}6.1 \\
1 \\
\end{array}$ & 2 & $\begin{array}{l}5797 \\
5 \\
\end{array}$ & $\begin{array}{l}9.9 \\
6 \\
\end{array}$ & \begin{tabular}{l|}
5.3 \\
1 \\
\end{tabular} & 4 & $\begin{array}{l}6038 \\
1 \\
\end{array}$ & $\begin{array}{l}7.3 \\
0 \\
\end{array}$ & $\begin{array}{l}0.2 \\
0 \\
\end{array}$ & 3 & $\begin{array}{l}7385 \\
0 \\
\end{array}$ & $\begin{array}{l}70 \\
33 \\
\end{array}$ & $\begin{array}{l}2.0 \\
3 \\
\end{array}$ & 3 & $\begin{array}{l}1097 \\
00\end{array}$ & \begin{tabular}{|l|}
8.9 \\
3 \\
\end{tabular} & $\begin{array}{l}4.0 \\
3 \\
\end{array}$ & 7 \\
\hline $\begin{array}{l}\text { Hailak } \\
\text { andi }\end{array}$ & $\begin{array}{l}186 \\
19 \\
\end{array}$ & $\begin{array}{l}2.3 \\
8 \\
\end{array}$ & $\begin{array}{l}8.5 \\
2 \\
\end{array}$ & 2 & $\begin{array}{l}2218 \\
0 \\
\end{array}$ & $\begin{array}{l}7.2 \\
1 \\
\end{array}$ & $\begin{array}{l}1.7 \\
7 \\
\end{array}$ & 2 & $\begin{array}{l}3413 \\
8 \\
\end{array}$ & $\begin{array}{l}7.6 \\
0 \\
\end{array}$ & $\begin{array}{l}2.1 \\
8 \\
\end{array}$ & 2 & $\begin{array}{l}4408 \\
5 \\
\end{array}$ & $\begin{array}{l}8.1 \\
2 \\
\end{array}$ & $\begin{array}{l}2.5 \\
9 \\
\end{array}$ & 3 & $\begin{array}{l}4814 \\
0 \\
\end{array}$ & $\begin{array}{l}7.3 \\
0 \\
\end{array}$ & $\begin{array}{l}0.8 \\
8 \\
\end{array}$ & 3 \\
\hline $\begin{array}{l}\text { Chira } \\
\text { ng }\end{array}$ & -- & & & & -- & & -- & & -- & & -- & & $\begin{array}{l}2500 \\
8\end{array}$ & & -- & & $\begin{array}{l}3533 \\
7\end{array}$ & $\begin{array}{l}7.3 \\
3\end{array}$ & $\begin{array}{l}3.5 \\
2\end{array}$ & 3 \\
\hline $\begin{array}{l}\text { Kamr } \\
\text { up }\end{array}$ & $\begin{array}{l}170 \\
278 \\
\end{array}$ & $\begin{array}{l}21 . \\
79\end{array}$ & $\begin{array}{l}13 . \\
42\end{array}$ & 8 & \begin{tabular}{|l|}
2506 \\
35 \\
\end{tabular} & $\begin{array}{l}20 . \\
77\end{array}$ & \begin{tabular}{|l|}
3.9 \\
4 \\
\end{tabular} & 9 & $\begin{array}{ll}6552 \\
15 \\
\end{array}$ & $\begin{array}{l}32 . \\
76\end{array}$ & $\begin{array}{l}4.9 \\
2 \\
\end{array}$ & 7 & $\begin{array}{l}5808 \\
1\end{array}$ & $\begin{array}{l}36 . \\
01\end{array}$ & $\begin{array}{l}3.3 \\
2 \\
\end{array}$ & 9 & $\begin{array}{l}1423 \\
94 \\
\end{array}$ & \begin{tabular}{|l|l|}
9.3 \\
8 \\
\end{tabular} & $\begin{array}{l}9.3 \\
8 \\
\end{array}$ & $\begin{array}{l}1 \\
6 \\
\end{array}$ \\
\hline $\begin{array}{l}\text { Kamr } \\
\text { up(M) }\end{array}$ & -- & & & & -- & & -- & & -- & & -- & & \begin{tabular}{|l|l}
8501 \\
36 \\
\end{tabular} & & & & $\begin{array}{l}1037 \\
011\end{array}$ & $\begin{array}{l}82 . \\
70\end{array}$ & $\begin{array}{l}2.0 \\
1 \\
\end{array}$ & $\begin{array}{l}1 \\
2\end{array}$ \\
\hline $\begin{array}{l}\text { Bongai } \\
\text { gaon }\end{array}$ & $\begin{array}{l}139 \\
90 \\
\end{array}$ & $\begin{array}{l}1.7 \\
9 \\
\end{array}$ & & 2 & $\begin{array}{l}3997 \\
9 \\
\end{array}$ & $\begin{array}{l}8.1 \\
5 \\
\end{array}$ & $\begin{array}{l}11 . \\
07\end{array}$ & 4 & $\begin{array}{l}7385 \\
4 \\
\end{array}$ & $\begin{array}{l}9.1 \\
5 \\
\end{array}$ & $\begin{array}{l}3.1 \\
2 \\
\end{array}$ & 5 & $\begin{array}{l}9722 \\
3 \\
\end{array}$ & $\begin{array}{l}12 . \\
13\end{array}$ & $\begin{array}{l}4.0 \\
4 \\
\end{array}$ & 5 & $\begin{array}{l}1098 \\
10\end{array}$ & $\begin{array}{l}14 . \\
86\end{array}$ & $\begin{array}{l}1.2 \\
2 \\
\end{array}$ & 5 \\
\hline $\begin{array}{l}\text { Nalbar } \\
\text { i }\end{array}$ & $\begin{array}{l}119 \\
04\end{array}$ & $\begin{array}{l}1.5 \\
2 \\
\end{array}$ & $\begin{array}{l}10 . \\
41\end{array}$ & 2 & $\begin{array}{l}1579 \\
2\end{array}$ & $\begin{array}{l}2.3 \\
2 \\
\end{array}$ & $\begin{array}{l}2.8 \\
7 \\
\end{array}$ & 2 & $\begin{array}{l}2347 \\
5 \\
\end{array}$ & $\begin{array}{l}2.3 \\
1 \\
\end{array}$ & $\begin{array}{l}2.0 \\
0 \\
\end{array}$ & 2 & \begin{tabular}{|l|}
2748 \\
6 \\
\end{tabular} & $\begin{array}{l}2.3 \\
9 \\
\end{array}$ & $\begin{array}{l}1.5 \\
9 \\
\end{array}$ & 2 & $\begin{array}{l}8273 \\
0 \\
\end{array}$ & $\begin{array}{l}10 . \\
72\end{array}$ & $\begin{array}{l}11 . \\
65\end{array}$ & $\begin{array}{l}1 \\
1\end{array}$ \\
\hline Baksa & $\begin{array}{l}--- \\
-- \\
-\end{array}$ & & & & $\begin{array}{l}- \\
- \\
\end{array}$ & & $\begin{array}{l}-- \\
-- \\
\end{array}$ & & $\begin{array}{l}- \\
- \\
\end{array}$ & & $\begin{array}{l}-- \\
-- \\
\end{array}$ & & $\begin{array}{l}- \\
-- \\
\end{array}$ & & & & $\begin{array}{l}1224 \\
2\end{array}$ & $\begin{array}{l}1.2 \\
9 \\
\end{array}$ & $\begin{array}{l}-- \\
-- \\
\end{array}$ & 2 \\
\hline $\begin{array}{l}\text { Darra } \\
\text { ng }\end{array}$ & $\begin{array}{l}197 \\
72\end{array}$ & $\begin{array}{l}2.5 \\
3 \\
\end{array}$ & $\begin{array}{l}18 . \\
66\end{array}$ & 3 & $\begin{array}{l}3217 \\
0\end{array}$ & \begin{tabular}{|l|}
3.8 \\
5 \\
\end{tabular} & $\begin{array}{l}5.0 \\
0 \\
\end{array}$ & 3 & \begin{tabular}{|l|l|}
6398 \\
4 \\
\end{tabular} & $\begin{array}{l}4.9 \\
2 \\
\end{array}$ & $\begin{array}{l}3.5 \\
0 \\
\end{array}$ & 4 & \begin{tabular}{|l|l|}
4170 \\
3 \\
\end{tabular} & $\begin{array}{l}4.9 \\
7 \\
\end{array}$ & $\begin{array}{l}1.5 \\
8 \\
\end{array}$ & 2 & $\begin{array}{l}5549 \\
4 \\
\end{array}$ & \begin{tabular}{|l|}
5.9 \\
8 \\
\end{tabular} & $\begin{array}{l}2.9 \\
0 \\
\end{array}$ & 3 \\
\hline $\begin{array}{l}\text { Udalg } \\
\text { uri }\end{array}$ & $\begin{array}{l}--- \\
-- \\
\end{array}$ & & & & $\begin{array}{l}---- \\
- \\
\end{array}$ & & $\begin{array}{l}-- \\
-- \\
\end{array}$ & & $\begin{array}{l}--- \\
- \\
\end{array}$ & & $\begin{array}{l}-- \\
-- \\
\end{array}$ & & \begin{tabular}{|l|}
3312 \\
5 \\
\end{tabular} & & & 2 & $\begin{array}{l}3757 \\
4 \\
\end{array}$ & $\begin{array}{l}4.5 \\
2 \\
\end{array}$ & $\begin{array}{l}1.2 \\
7 \\
\end{array}$ & 3 \\
\hline $\begin{array}{l}\text { ASSA } \\
\text { M }\end{array}$ & $\begin{array}{l}781 \\
288\end{array}$ & $\begin{array}{l}10 \\
0\end{array}$ & $\begin{array}{l}8.5 \\
2\end{array}$ & $\begin{array}{l}5 \\
3\end{array}$ & $\begin{array}{l}1289 \\
222\end{array}$ & $\begin{array}{l}10 \\
0\end{array}$ & $\begin{array}{l}5.1 \\
3\end{array}$ & $\begin{array}{l}7 \\
2\end{array}$ & \begin{tabular}{|l|}
2487 \\
795
\end{tabular} & $\begin{array}{l}\text { 10 } \\
0\end{array}$ & $\begin{array}{l}3.3 \\
4\end{array}$ & $\begin{array}{l}9 \\
3\end{array}$ & \begin{tabular}{|l|l|}
3439 \\
249
\end{tabular} & $\begin{array}{l}\text { 10 } \\
0\end{array}$ & $\begin{array}{l}3.2 \\
9\end{array}$ & $\begin{array}{l}1 \\
2 \\
5\end{array}$ & $\begin{array}{l}4398 \\
542\end{array}$ & $\begin{array}{l}10 \\
0\end{array}$ & $\begin{array}{l}2.4 \\
9\end{array}$ & $\begin{array}{l}2 \\
1 \\
4\end{array}$ \\
\hline
\end{tabular}

Source : Computed by the researcher himself.

Columns indicate: I= Urban Population; II= Urban population (\%); III= Annual Growth Rate (\%); IV= Total Urban Centre

Nevertheless the spatial distribution and growth of urban population in Assam is not so homogeneous as the case above. Since 1901 till 1951 the districts of Kamrup and Nagaon recorded the largest urban population concentration with somewhat equal number of urban centres as well as urban dwellers. But after 1951, Kamrup with a record annual growth rate of 13.42 per cent registered nearly four times increase in its urban population from 48,321 in 1951 to 1,70,278 in 1961. Following the suit, districts like Tinsukia(13.39\%), Lakhimpur(12.19\%), Golaghat(10.51\%) and Nalbari(10.41\%) experienced very high annual growth rates during the same time period. Besides the general factors like natural increase in urban population, urban area or declaration of new urban centres, the main reason for such rapid increase in urban population is unprecedented volume of immigration especially of the Bengali Hindus and Muslim peasants from erstwhile East Pakistan. The Bengali Hindus from East Pakistan continued to pour in because of recurrent communal disturbances there. Thus urban centres being more accessible and presence of ample opportunities for income generation becomes easy destination of the immigrants.

However surprisingly after 1961, the urban population experienced slow growth rate with only one district i.e. Bongaigaon recording more than 10 per cent annual growth rate. Infact Darrang which registered the highest annual growth rate of urban population in 1961 (18.66\%) experienced only 5 per cent growth in 1971. Gradually in 2001 no district except Dhemaji recorded more than 6 per cent annual growth rate. In the subsequent census year Nalbari registered the highest growth rate of 11.65 per cent which is a direct outcome of the recognition of 9 rural service centres as urban units. Contrary to this scenario, although the number of urban centres increased from 6 to 8 during the last decade (2001-2011) in Sonitpur, it registered a negative growth rate of -0.11 per cent. The main basis for this negative growth is due to the decrease in the overall urban population of Tezpur-the premier city of Sonitpur and Rangapara. The Barika Chuburi which was an outgrowth of Tezpur in 2001 was recognized as a separate urban centre in 2011. Therefore devoid of its area and population Tezpur's aggregate urban population declined. Moreover there has been an increase in the number of male migrants from Tezpur and Rangapara. Infact all the wards of Rangapara showed less urban male population as compared to 2001 census. Consequently these factors have led to low natural increase in urban population in the region.

\subsection{Spatial Pattern of Urban Intensity and Level of Urbanisation in Assam, 1991-2011}

Urban Intensity has been computed to show the meso-level regional variation in the distribution of urban population in Assam. Similarly the level of Urbanisation has been computed which show some spatial variation between the two indices as well as within the indices when analysed in temporal context. The level of urban intensity in 1991 is highest in Cachar closely followed by Dibrugarh and Dhubri with an aggregate average score of more than 60 per cent (Table 2). Conversely the districts of Nalbari, Jorhat, Dima Hasao, Sibsagar and Karbi Anglong experienced aggregate score less than 20 per cent. Similar trends have been observed in the subsequent years also. 
Urban Myth and Reality: The State of Urban Growth and Development In Assam

TABLE 2: Spatial variation in the Level of Urbanisation and Urban Intensity in Assam, 1991-2011

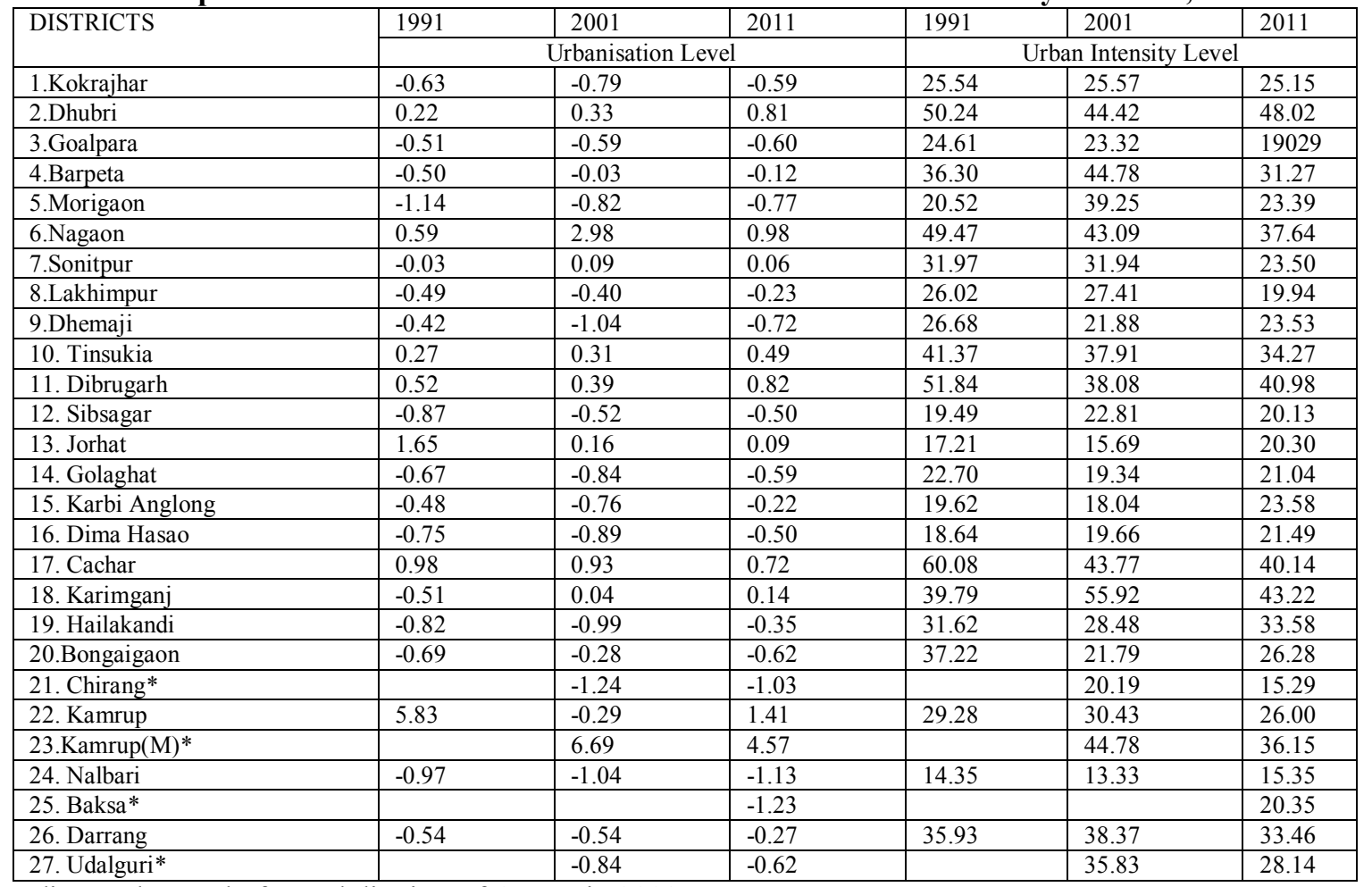

* Indicates the newly formed districts of Assam in 2003.

Nevertheless, the PCA scores for urbanisation level reveal somewhat contradictory scenario when compared with the urban intensity of the region. Urbanisation level is highest for the district of Kamrup in 1991 followed by Jorhat, Cachar and Nagaon. On the contrary, the districts of Morigaon, Nalbari and Sibsagar registered lowest levels of Urbanisation with composite scores below -0.8 per cent respectively. However with the division of the premier city of entire North-East India alone contains more than 22 per cent of the total urban population of Assam. On the other hand, Chirang witnessed the lowest level of urbanisation accompanied by Dhemaji, Nalbari, Hailakandi and the hill district of Dima Hasao with negative composite value. Infact the combined proportion of urban population in all these districts is only 6.28 per cent.

Thus although some similarity is seen in the spatial variation in level of urbanisation and urban intensity in the state, but there exist some exceptions. For instance, the level of urbanisation was highest in Kamrup in 1991 but the urban intensity was among the lowest. The main cause of high urbanisation level is its highest percentage of urban population whereas the low urban density is the root cause of low urban intensity. Similar is the case with Jorhat, which experienced second most urbanised district with respect to urbanisation level but lowest in terms of urban intensity. Less number of urban centres coupled with low urban population has pushed it to this level. However in subsequent years, the urban intensity scenario of Kamrup(M) is at par with the urbanisation level including the other newly carved out districts of Chirang, Baksa and Udalguri. 

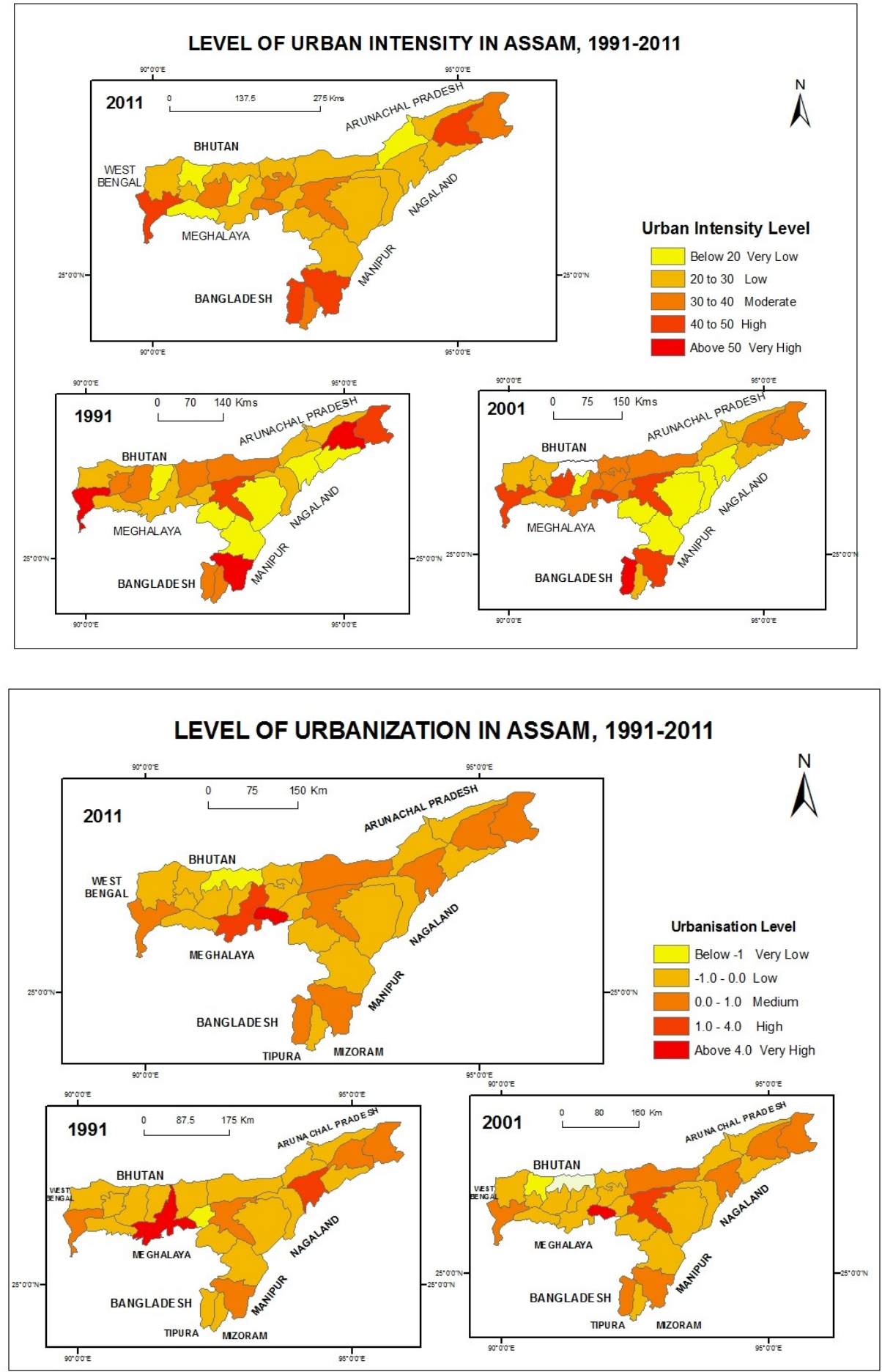

\subsection{Spatio-Temporal Variation in Level of Urban Development in Assam, 1991-2011}

From the previous discussions it is clear that there exist sharp regional variation either in case of urbanisation level or urban intensity although they both sound similar. However the main point of concern is that whether the urbanisation in the state is due to urban development or whether the course of urbanisation has preceded the urban development process. A glimpse at the table 3 reveals that since 1991 till date Kamrup and latter on divided Kamrup experienced the highest level of urban development with very high composite value. The other districts which have experienced significant urban development include Dibrugarh, Sibsagar, Cachar, Jorhat and Tinsukia. Similarly the under developed districts include Karbi Anglong, Dhubri, Baksa, Goalpara, Dhemaji and Chirang. 
TABLE 3: District wise Level of Urban Development in Assam, 1991-2011

\begin{tabular}{|c|c|c|c|}
\hline DISTRICTS & 1991 & 2001 & 2011 \\
\hline 1.Kokrajhar & -3.49 & -3.48 & -1.10 \\
\hline 2.Dhubri & -4.53 & -6.14 & -5.42 \\
\hline 3.Goalpara & -5.43 & -4.74 & -6.98 \\
\hline 4.Barpeta & -3.02 & -1.35 & -0.33 \\
\hline 5.Morigaon & -5.18 & -4.21 & -3.26 \\
\hline 6.Nagaon & 0.87 & 1.46 & 2.90 \\
\hline 7.Sonitpur & 3.27 & 2.69 & 3.56 \\
\hline 8.Lakhimpur & -2.69 & -2.10 & -0.75 \\
\hline 9.Dhemaji & -3.89 & -5.25 & -3.90 \\
\hline 10. Tinsukia & 3.18 & 3.18 & 3.28 \\
\hline 11. Dibrugarh & 6.09 & 5.19 & 4.73 \\
\hline 12. Sibsagar & 4.01 & 2.96 & 3.16 \\
\hline 13. Jorhat & -0.14 & 2.95 & 5.29 \\
\hline 14. Golaghat & 2.91 & 0.94 & 2.64 \\
\hline 15. Karbi Anglong & -6.33 & -4.04 & -2.36 \\
\hline 16. Dima Hasao & -3.54 & -0.94 & 1.99 \\
\hline 17. Cachar & 3.75 & 3.04 & 2.98 \\
\hline 18. Karimganj & 4.76 & 3.13 & 2.21 \\
\hline 19. Hailakandi & 1.20 & 0.89 & 2.55 \\
\hline 20.Bongaigaon & 1.24 & 0.22 & -6.14 \\
\hline 21. Chirang* & & & -6.14 \\
\hline 22. Kamrup & 9.91 & 9.00 & -2.74 \\
\hline 23.Kamrup(M)* & & & 14.95 \\
\hline 24. Nalbari & -1.77 & -0.02 & -2.54 \\
\hline 25. Baksa* & & & -9.16 \\
\hline 26. Darrang & -1.16 & -3.37 & -3.94 \\
\hline 27. Udalguri* & & & -2.42 \\
\hline
\end{tabular}

* Indicates the newly formed districts of Assam in 2003.

Thus a meso-level analysis shows that there exist a close positive relationship between urbanisation level, urban intensity and urban development in the region. However some administrative units show a negative relationship too. For instance, the level of urbanisation and urban intensity is very high at Dhubri, but it ranks miserably with respect to urban development. Infact in the year 2001, Dhubri was the least developed district of Assam. Similar situation is been observed in the districts of Nagaon and divided Kamrup. Although both the districts have huge urban population but the urban centres of these districts lacks the basic amenities required for a decent urban living. Thus these two districts are only experiencing massive urban population growth but the urban dwellers lack minimum standard of living. However contrary to this view, there also exist some districts where urban development precedes urban population growth and urbanisation level. Districts of Sibsagar, Dima Hasao, Golaghat and Bongaigaon although are experiencing low levels of urbanisation but they fare well with respect to urban development.

Among the districts, Jorhat, Tinsukia and Nagaon have shown positive trends in urban development since 1991, whereas the districts of Karimganj, Darrang, Cachar and Dibrugarh are constantly showing declining trends. The main cause of the decrease in the urban development scenario is the addition of many new census towns to the urban setting of these districts based primarily on population considerations while negating the availability of any basic urban amenity in the urban centre. Thus urban development follows urbanisation process in Assam.

\section{Urban Development Regimes}

Based upon the composite values of urban development, Assam can be sub-divided into 5 urban development zones. They are:

i) Dynamic Region- The district of erstwhile Kamrup and at present Kamrup(M) recorded the highest level of urban development followed by Jorhat with composite value above 5. Kamrup(M) being the seat of the state's capital Dispur and the largest urban centre of entire North-East i.e. Guwahati recorded the highest composite value of 14.95. Dispur being the administrative centre and Guwahati being the premier city are the first destination of any developmental project including infrastructural and institutional projects. Till 2001, undivided Kamrup experienced low urban development as compared to the divided Kamrup(M), as most of the urban areas were included into it. Contrary to this scenario the other Kamrup experienced negative rate of urban development as most of the rural areas were incorporated into it. Similarly Jorhat shows an interesting positive trend with respect to urban development since 1991. In 1991 she experienced negative rate of urban development (-0.14) which gradually increased to 2.95 in 2001 and further increased to 5.27 in the year 2011 . Infact Jorhat witnessed an increase in almost all the considered variables securing second most literate district with third highest number of educational and medical institutions. 
ii) Prospective Region- The districts of Dibrugarh, Sonitpur, Tinsukia and Sibsagar with composite value ranging from 3 to 5 , follows the traits of dynamism qualifying for recognition as prospective region. All these districts contain high share of urban literates (above 85\%) with sanitation (latrines) and LPG (cooking fuel) services available to more than 93 and 70 per cent urban households respectively. Moreover most of the urban centres of these districts are either industrial or commercial or transport hub which renders it more prone to any developmental aspects. Some of these urban centres are Namrup (fertilizer and natural gas), Naharkatia and Duliajan (Oil and natural gas), Moran and Chabua (tea) of Dibrugarh districts; Dhekiajuli (tea), Gohpur (transport hub to Itanagar) and Tezpur (largest urban centre of North bank of Brahmaputra) of Sonitpur district; Digboi (oil refinery), Doom Dooma, Margherita and Ledo (coal, tea and plywood) including the largest commercial centre of eastern Assam - Tinsukia of Tinsukia district and; Nazira (oil), Simaluguri and Amguri (railway towns) including the historic town of Sibsagar of Sibsagar district. The prospect of this region lies in the availability of mineral resources like coal, oil and natural gas and associated trend of industrialization and socio-cultural dominance of Sibsagar and Tezpur UA.

iii) Developing Region- Cachar, Nagaon, Golaghat, hailakandi, Karimganj, Dima Hasao and Bongaigaon are the districts which together combine to form the developing zone where the developmental process is very modest. It is worth mentioning that although these administrative units collectively possesses more than 25 per cent of the total urban population but their developmental process is very slow with composite value ranging from 0 to 3. Besides Silchar UA, Nagaon is the only Class I city of the region, where development of urban amenities have cropped in. Silchar being the commercial and transport centre of southern Assam have helped in elevating the urban development scenario of Cachar. Bongaigaon, BRPL Township, Badarpur, Haflong, Umrangsu, HPC Township, Hojai, Lumding etc are some of the other important urban centres of this region. The main cause for such a fragile state of urban development is the recognition of 23 new rural service centres as census towns to the existing urban scenario of the region. These new urban centres desperately lack the basic urban amenities which is essential for a decent standard of living.

iv) Stagnant Region- This region comprises of the districts of Barpeta, Lakhimpur, Kokrajhar, Karbi Anglong, Udalguri, Nalbari, Kamrup, Morigaon, Dhemaji and Darrang with negative urban development scores (0 to -5). Although this region is the largest of all other regions, but it contains only 19 per cent of the total urban population of the state where developmental process is at infant stage. Most of the urban centres are large rural service centres where most of the services like availability of tap water, LPG as cooking fuel, electricity, and sanitation services etc. to the urban households are comparatively low. Except North Lakhimpur which is a Class II category urban centre, all other towns of the region are smaller in size with regard to both population and area where development process have just initiated. Addition of 27 new urban centres is another reason for its under developed nature. Barpeta, Lakhimpur, Nalbari, Morigaon and Dhemaji are agricultural based districts whereas Kokrajhar, Udalguri and Kamrup are newly carved out states where rural areas predominate.

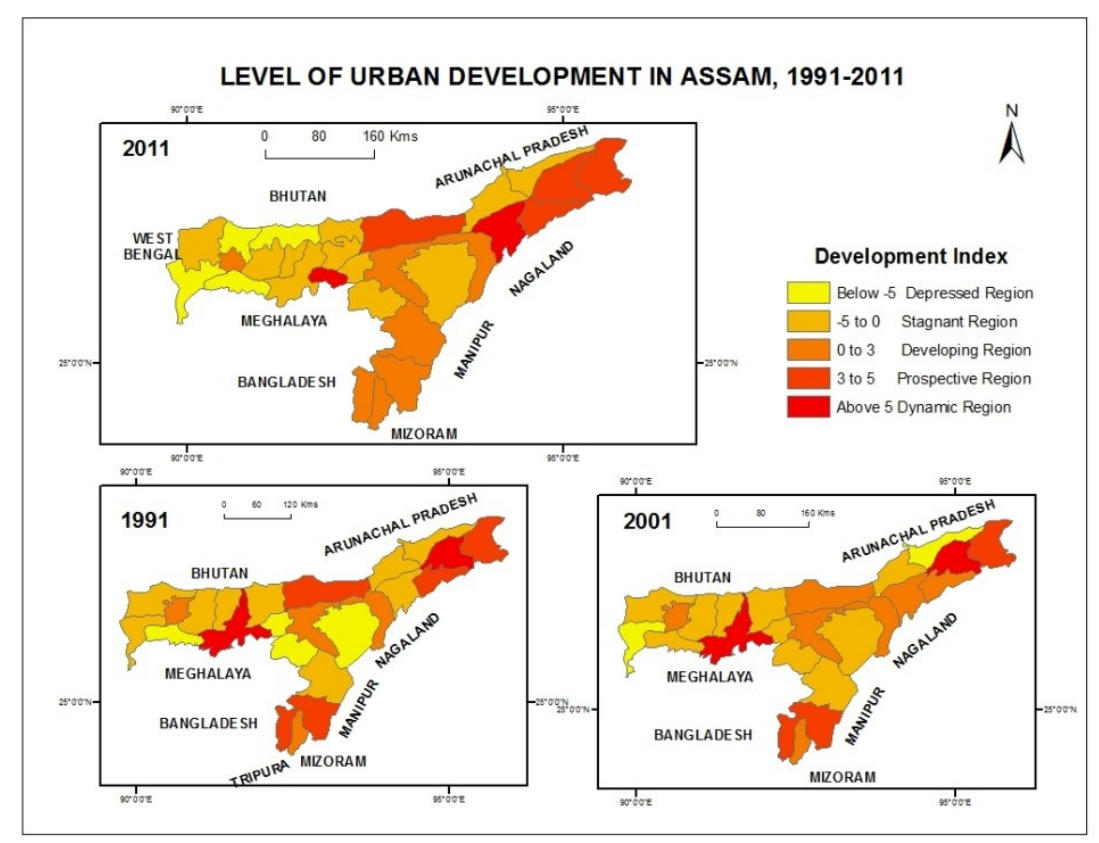

v) Depressed Region- The districts of Dhubri, Chirang, Goalpara and Baksa collectively form the least urban developed region with highly negative (Below -5) composite value. It is worth mentioning that although Dhubri alone possesses about 5 per cent of the total urban population but it is among the least developed district of the 
state. The districts of Chirang and Baksa are newly carved out states where urbanisation and urban development process have just initiated. With very low level of literacy rate along with minimal availability of the considered amenities, these districts are the most under developed districts of Assam.

\section{Conclusion}

The foregoing discussion reveals that Assam as a whole experienced high urban growth rate basically due to rural-urban migration. However the addition of 89 new urban centres to the urban scenario of the state has also contributed to higher urbanisation levels but low level of urban development. Within the region again, the urbanization and urban development level has been higher for the districts of $\operatorname{Kamrup}(\mathrm{M})$, Dibrugarh, Tinsukia and Cachar. However some districts like Dhubri and Sibsagar show a negative correlation.

The causes behind prevailing spatial variation in urbanisation level and urban development in the region have become more clear when the pattern of urban growth rate and urban development is analysed separately considering as many as ten development indicators ranging from the period 1991 till 2011. Thus it has become clear that with the declaration of more and more new urban centres further degrade the development scenario of the region. Consequently urbanisation today is viewed as a problem rather than an important indication of regional development. Accordingly grass root development approach to rural service centres must be adopted by the administration so that the mass exodus of rural population to cities can be minimized. Moreover the present population based criteria for declaration of any rural unit as town must be avoided as far as practicable. Simultaneously the existing urban centres must also be developed so that the after effects of development trickle down to its hinterland.

\section{References}

[1]. Betal, H.R., Use of Statistical Techniques to study Urbanisation in West Bengal, Geographical Review of India, 63(3), 2001, 258276.

[2]. Bogue, D.J. and Zachariah, K.C., Urbanisation and Migration in India, in Turner, Roy. (Ed.), India's Urban Future, Oxford University Press, Bombay, (1962), 27-54.

[3]. Borah, J., Process of Urbanisation in Assam, North Eastern Geographer, 22, (1 \& 2), 1990, 22-23.

[4]. Cherunilam, F., Urbanisation in Developing Countries: A Socio-economic and Demographic Analysis, (Himalaya Publishing House, Bombay, 1984).

[5]. Clyde Mitchell-Weaver., Urban Systems Theory and Third World Development: A Review, 13(4), 1991, 419-441.

[6]. D'souza, V.S., Urban Development in India: Demographic, Functional and Socio-cultural Perspectives, in P.K. Muttagi (Ed.), Urban Development-A Perspective, Tata Institute of Social Sciences, Bombay, (1976), 52-58.

[7]. Johnson, James, H., Urban Geography: An Introductory Analysis, (Pargamon Press, London, 1977).

[8]. Kaushik, S., Trends of Urbanisation: Some insights from the Census, Geographical Review of India, 65 (2), $2003,181-188$.

[9]. Kingsley, D., Urbanisation and Urban Systems in India (Oxford University Press, New Delhi, 1962).

[10]. Manta, R., et al., Urbanisation and Growth of Small Towns in Assam, India, in www.unil.ch/webdav/site/iguurban/shared/Manta.pdf.

[11]. Nagpaul, H., Modernization and Urbanisation in India: Problems and Issues (Rawat Publications, Jaipur, 1996).

[12]. Prakasa Rao, V.L.S., Urbanisation in India: Spatial Dimensions (Concept Publishers, New Delhi, 1983, 24-43).

[13]. Prasad, B.K., Urban Development (Sarup and Sons Publications, New Delhi, 2003, 34-52).

[14]. Ramachandran,R., Urbanisation and Urban Systems in India (Oxford University Press, New Delhi, 1996).

[15]. Sharma, H.N., Pattern of Urbanisation in Assam: A Geographical Analysis, North Eastern geographer, 2(1), 1970, 83-94.

[16]. Sharma, H.N., Urban Growth and Spatial Pattern of Urban Development in the Brahmaputra Valley, Assam, North Eastern Geographer, 11(1\&2), 1979, 28-47.

[17]. Sharma, N., Measurement of Unevenness in Distribution of Urban Population in Bihar: An Approach to Regionalisation, North Eastern Geographer, 10(1\&2), 1978, 23-32

[18]. Shukla, P., Population Spectrum in the Light of Urbanisation in Sagar Dristrict, During 1991-2001, Geographical Review of India, 69(1), 2007, 33-38.

[19]. Stafford, J., Demographic Correlates of Urban Development, Canadian Studies in Population, 7, 1980, 67-80.

[20]. Census of India, Tables on Houses, Households Amenities and Assets, Series-4, Part VII, Assam, 1991.

[21]. Census of India, Tables on Houses, Households Amenities and Assets, Series-19, Assam, 2001 and 2011.

[22]. Census of India, Town Directory of Assam, 1991 and 2001 